by PCR, sequenced and analysed for drug resistance mutations and subtype information.

Results HIV-2 RNA was detected in 7 of 10 ART-naïve and 2 of 6 ART-experienced patients. Detectable HIV-2 viral loads in these patients ranged from below the lower limit of quantification $(<2.35 \mathrm{log} \mathrm{IU} / \mathrm{ml})$ to $5.45 \mathrm{log} \mathrm{IU} / \mathrm{ml}$. One ART-experienced patient had M184V, K65R and Y115F mutations in RT sequences from both plasma and PBMC. There were no drug resistance mutations identified from ART- naïve samples.

Conclusion This is the first study in Ghana to show evidence of mutations in HIV-2 strains from patients receiving HIV-1 targeted antiretrovirals. The results prompt monitoring of drug resistance to improve clinical management of HIV-2 infected patients.

\section{LB 2.59 IMPROVING STD SCREENING IN HIV CARE THROUGH IMPLEMENTATION OF SELF-COLLECTED EXTRAGENITAL SWABS}

${ }^{1}$ Ina Park, ${ }^{2}$ Jeffrey Schapiro, ${ }^{3}$ Leo Hurley, ${ }^{4} \mathrm{C}$ Brad Hare, ${ }^{5}$ Sally Slome, ${ }^{6}$ Jason Flamm, ${ }^{7}$ Lauren Nelson, ${ }^{5}$ Michael Allerton. ${ }^{1}$ University of California San Francisco, San Francisco, USA; ${ }^{2}$ Kaiser Permanente Northern California Regional Laboratory, Berkeley, CA, USA; ${ }^{3}$ Kaiser Permanente Division of Research, Oakland, CA, USA; ${ }^{4}$ The Permanente Medical Group, San Francisco, USA; ${ }^{5}$ The Permanente Medical Group, Oakland, CA, USA; ${ }^{6}$ The Permanente Medical Group, Sacramento, CA, USA; ${ }^{7}$ California Department of Public Health, STD Control Branch, Richmond, CA, USA

\subsection{6/sextrans-2017-053264.235}

Introduction Screening for syphilis, gonorrhoea (GC) and chlamydia (CT) is recommended at least annually for HIV-positive men who have sex with men (MSM) in the United States (US). Recent analyses from the US Medical Monitoring Project demonstrate that STD screening of HIV-positive MSM remains far below that recommended by guidelines; specific data on extragenital GC/CT screening is not reported. We implemented a quality improvement intervention to improve STD screening (syphilis, GC/CT) in a large managed care organisation (16 centres) including didactic training and implementation of selfcollected swabs for GC/CT.

Methods We analysed data from the Kaiser Permanente Northern California HIV Registry to calculate the proportion of MSM tested for syphilis and GC/CT (any site, rectal/pharyngeal site) at least once in the prior year. Laboratory validation of self-collected swabs was completed by $1 / 2014$, rolled out at five centres by $12 / 2014$, and 11 centres by $11 / 2016$. Screening data were finalised for analysis in 1/2017. Three time periods were examined: baseline (6/2012), 1 year (11/2015), and 2 years (11/2016) post initial implementation of self-collection. Cochran-Armitage was used to test for trends.

Results During the study period, the denominator of eligible HIV-positive MSM increased from $n=4499$ to 5866. Annual screening for GC/CT (any site) significantly increased from $45.2 \%$ to $58.3 \%$ ( $\mathrm{p}_{\text {trend }}<0.0001$ ); extragenital GC/CT (among those screened) increased from $48.4 \%$ to $58.1 \%$ ( $\left.\mathrm{p}_{\text {trend }}<0.0001\right)$. Medical centres that implemented self-collected swabs within the first year reported higher extragenital screening rates than those who did not $(60.6 \%$ vs $20.2 \%$, $\mathrm{p}<0.0001$ ), this difference persisted into year 2. Syphilis screening also increased from $73.6 \%$ to $76.8 \%$ $\left(p_{\text {trend }}=0.0002\right)$.

Conclusion Implementation of self-collected GC/CT swabs is an effective intervention to increase STD screening among MSM in a large US managed care organisation. This intervention should be disseminated to other settings to improve currently suboptimal STD screening rates among MSM.

\section{LB2.60 FIELD EVALUATION OF A NOVEL DUAL HIV/SYPHILIS RAPID TEST - MALAWI, 2014-2015}

${ }^{1}$ Virginia Bowen, ${ }^{1}$ Hetal Patel, ${ }^{1}$ Kathryn Lupoli, ${ }^{2}$ Geoffrey Chipungu, ${ }^{1}$ Bharat Parekh, ${ }^{1}$ Cheng Chen, ${ }^{3}$ Benson Chilima, ${ }^{3}$ Frank Chimbwandira, ${ }^{1}$ Damien Danavall, ${ }^{1}$ Vedapuri Shanmugam, ${ }^{4}$ Enid Mfungwe, ${ }^{1}$ Janell Routh, ${ }^{1}$ Mary Kamb. ${ }^{1}$ Centres for Disease Control And Prevention (CDC), Atlanta, USA; ${ }^{2}$ Centres for Disease Control And Prevention $(C D C)$, Lilongwe - Malawi; ${ }^{3}$ Ministry of Health, Lilongwe - Malawi; ${ }^{4}$ Ssi-Malawi, Lilongwe - Malawi

\subsection{6/sextrans-2017-053264.236}

Introduction Dual HIV/syphilis rapid diagnostic tests (RDTs) may prevent congenital syphilis by facilitating syphilis diagnosis in pregnant women receiving HIV testing. The dual HIV-1/ 2 treponemal syphilis RDT (Chembio DPP HIV-Syphilis Assay) performs well in the lab, but its field performance is unknown. We investigated test performance under field conditions for this dual RDT and Malawi's single RDTs for HIV and syphilis to assess whether the dual RDT might be a suitable substitute for the first-line single RDT in Malawi's HIV algorithm.

Methods During Jul 2014-Nov 2015, 1798 pregnant women attending a first antenatal visit were recruited if their HIV status was negative or unknown. Women received the single HIV (Determine HIV-1/2) and syphilis (Determine Syphilis TP) RDTs and the dual RDT. By Dec 2016, CDC had performed Rapid Plasma Reagin (RPR), Treponema pallidum particle agglutination (TPPA), and $3^{\text {rd }}$-generation HIV EIA testing with Western Blot confirmation. In Jan 2017, the validity of all RDTs relative to the CDC HIV algorithm, TPPA, and TPPA/ RPR results were calculated.

Results Of 1791 women (99.6\%) with complete results, 258 (14.4\%) were HIV-positive by CDC's algorithm; 81 (4.5\%) were TPPA+; and 46 (2.6\%) were TPPA+/RPR+. The dual RDT was $95.0 \%$ sensitive and $96.0 \%$ specific for HIV; the single HIV RDT was $93.0 \%$ sensitive and $99.3 \%$ specific. HIV test specificities were significantly different $(p<0.01)$. Both dual and single HIV RDTs were 96.9\% sensitive during repeat lab testing. Using TPPA+ as the standard, the dual RDT was $69.1 \%$ sensitive and $99.8 \%$ specific for syphilis; the single syphilis RDT was $63.0 \%$ sensitive and $99.8 \%$ specific. Among women most likely to vertically transmit syphilis (TPPA+/RPR + , titer $\geq 1: 4$ ), the dual and single RDTs were $100.0 \%$ and $88.2 \%$ sensitive, respectively.

Conclusion The dual RDT syphilis component performed comparably to the single syphilis RDT and performed very well among women likely to vertically transmit syphilis. The dual RDT HIV component had comparable sensitivity but lower specificity than the single HIV RDT.

\section{LB2.61 NEAR FULL LENGTH DEEP SEQUENCING OF NEWLY ACQUIRED HIV INFECTIONS IN SAN FRANCISCO}

${ }^{1} \mathrm{R}$ Hance, ${ }^{1} \mathrm{~T}$ Notton, ${ }^{2} \mathrm{H}$ Truong, ${ }^{1,2} \mathrm{R}$ Grant. ${ }^{1}$ Gladstone Institute of Virology and Immunology, USA; ${ }^{2}$ University of California San Francisco, USA

10.1136/sextrans-2017-053264.237 